\title{
Does Continuous Sciatic Nerve Block Provide Superior Pain Control to Opioids in Lower Limb Amputation Patients? A Retrospective Study.
}

\author{
Dr. Venkatesan Duraiswamy (ST7 Trainee), Dr. A. Malik (Cons), Dr. A.Krol (Cons) \\ St. George's University Hospital, London, UK
}

\section{Background and Aims:}

Postoperative stump pain after major lower limb amputation is a significant impediment to the recovery of amputees. Continuous perineural infusions of local anaesthetic are a safe and effective method for reducing post-amputation opioid analgesic medications after major lower limb amputation. The aim of the study is to compare the postoperative pain scores between the continuous sciatic nerve block (CSNB) group and without the nerve catheter group.

\section{Methods:}

All data was collected from patient notes held in Medical Records and Electronic document management system. Retrospective data was collected from the nerve block local anaesthetic infusion chart, anaesthetic chart, drug chart and surgical care pathway. We compared this data with our previous audit done in 2016. We included 41 patients in the sciatic catheter group and 43 patients in the non-catheter group. All the patients in the non-catheter group received PCA morphine or strong oral opioid in the postoperative period. Pain scores were analysed based on a numerical rating scale of $0-4(0=$ no pain at rest/on movement, $1=$ mild pain on movement, $2=$ moderate pain on movement, $3=$ severe pain on movement and $4=$ continuous pain at rest/severe pain on movement).

\section{Results:}

In the CSNB group $86 \%$ of the patients had a pain score between 0 to 2 whereas in the Non- CSNB $72.5 \%$ of patients were having pain score between 0 to 2. In the CSNB group, $14 \%$ of the patients had a pain score between 3 to 4 , whereas in the Non-CSNB group $27.5 \%$ of patients had a pain score between 3 to 4 (figure 1). When we looked into patients who had the pain score of 0 , CSNB group had $68.3 \%$ of patients whereas Non- CSNB group had $32.2 \%$ of patients which is statistically significant with a $P$ value of $<0.05$ (figure 2). In the CSNB group, $84.1 \%$ had PCA morphine or strong oral opioids, as mentioned earlier all the patients in the Non-CSNB group had PCA morphine or strong oral opioids.
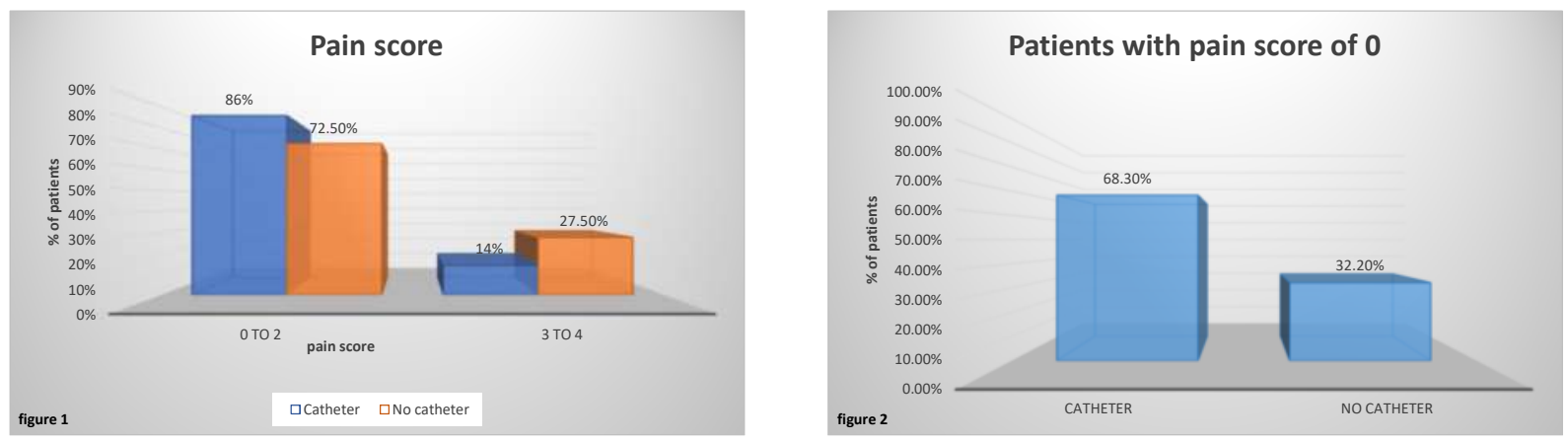

\section{Conclusion:}

This study shows patients managed with continuous sciatic nerve block had better pain relief in the postoperative period and better patient satisfaction and experience during their hospital stay. There are only a few randomised control trials available regarding the use of CSNB in this cohort and many studies failed for various reasons. Therefore, we are planning to do a prospective randomised control trial to assess the efficacy of CSNB, requirements of postoperative opioids \& their side effects and the incidence of phantom pain.

\section{References:}

1. Malik A, Krol A Postoperative pain management in patients presenting for lower limb amputation. Audit of current practice in tertiary referral centre . ESRA7-0211, Reg Anesth Pain Med, Vol 42, N 5, suppl 1, Sep- Oct 2017

2. National Confidential Enquiry into Patient Outcome and Death, Lower Limb Amputation: Working Together, November 2014.

\section{Funding and Competing interest:}

None. 